\title{
Hypothetical role of large nuclear gravity in understanding the significance and applications of the strong coupling constant
}

\author{
U.V.S. Seshavatharam ${ }^{1}$ and S. Lakshminarayana ${ }^{2}$ \\ ${ }^{1}$ Honorary faculty, I-SERVE, Survey no-42, Hitech city, Hyderabad-84,Telangana, INDIA \\ 2 Dept. of Nuclear Physics, Andhra University, Visakhapatnam-03,AP, INDIA \\ Emails: seshavatharam.uvs@gmail.com (and) 1nsrirama@gmail.com \\ Orcid numbers : 0000-0002-1695-6037 (and) 0000-0002-8923-772X
}

\begin{abstract}
As there exist no repulsive forces in strong interaction, in a hypothetical approach, strong interaction can be assumed to be equivalent to a large gravitational coupling. Based on this concept, strong coupling constant can be defined as a ratio of the electromagnetic force and the gravitational force associated with proton, neutron, up quark and down quark. With respect to the product of strong coupling constant and fine structure ratio, we review our recently proposed two semi empirical relations and coefficients 0.00189 and 0.00642 connected with nuclear stability and binding energy. We wish to emphasize that- by classifying nucleons as 'free nucleons' and 'active nucleons', nuclear binding energy can be fitted with a new class of 'three term' formula having one unique energy coefficient. In table-3, we present the estimated nuclear binding energy data for $Z=3$ to 120 and compare it with the two standard semi empirical mass formulae as a supplementary file.
\end{abstract}

Key words: Large nuclear gravity, Strong coupling constant; nuclear stability; nuclear binding energy; Free nucleons; Active nucleons;

\section{Introduction}

With reference to nuclear stability and binding energy, relationship between nuclear force and strong force is still a grey area and is a challenging task for field experts and young scientists [1,2]. It is well established that,

1) Less than $0.8 \mathrm{fm}$, strong force is mediated by gluons.

2) At a range of 1 to $3 \mathrm{fm}$, strong force is mediated by mesons.

3) Neutrons, protons, baryons and mesons are made up of quarks.

4) Gluons interact with quarks and other gluons and mesons interact with neutrons and protons.

5) Strong force that binds quarks into neutrons, and protons can be called as 'residual strong force' or simply 'nuclear force'.

6) Within the quark surroundings, force is strong and distance independent.

7) Nuclear force is weaker and rapidly decreases with increasing distance among nucleons (bound quarks).

8) Even though nuclear force is weaker than the strong force, still it is very energetic in producing gamma rays and holding nucleons with large binding energy.

9) Strength of strong interaction is parameterized by strong coupling constant, $\alpha_{s} \cong 0.1181$ [3].
In this context, in this paper, with reference to earlier published concept of large nuclear gravitational coupling [5,6,7,8] and our recent paper [9] and references therein, we make an attempt to understand the physical significance and applications of strong coupling constant with respect to nuclear stability and binding energy.

\section{To understand the physical significance of strong coupling constant}

Strong coupling constant $\left(\alpha_{s}\right)$ can be defined as a ratio of the electromagnetic force and the gravitational force associated with proton, neutron, up quark and down quark. Mathematically, it can be represented as

$$
\alpha_{s} \cong \frac{e^{2}}{4 \pi \varepsilon_{0}}\left[\begin{array}{l}
{\left[G_{s} m_{p}\left(2 m_{u}+m_{d}\right)\right]} \\
+\left[G_{s} m_{n}\left(m_{u}+2 m_{d}\right)\right]
\end{array}\right]^{-1}
$$

where $G_{s}=$ large nuclear gravitational constant, $\left(m_{p}, m_{n}\right)=$ Proton and neutron masses and $\left(m_{u}, m_{d}\right)=\mathrm{Up}$ and down quark masses. 
In our earlier published papers, we proposed that [4], $G_{s} \cong 3.329561 \times 10^{28} \mathrm{~m}^{3} \mathrm{~kg}^{-1} \mathrm{sec}^{-2}$. With reference to particle data group [3],

$$
\left.\begin{array}{l}
m_{u} \cong 2.16_{-0.26}^{+0.49} \mathrm{MeV} \\
m_{d} \cong 4.67_{-0.17}^{+0.48} \mathrm{MeV}
\end{array}\right\}
$$

Based on these values, estimated $\alpha_{s} \cong 0.11333$ and its recommended world average $\alpha_{s} \cong 0.1181$. By refining the magnitudes of up and down quark masses and the large nuclear gravitational constant, absolute value of $\alpha_{s}$ can be fixed. Conceptually, it seems better to understand that,

$$
\frac{e^{2}}{4 \pi \varepsilon_{0}} \cong \alpha_{s}\left\{\begin{array}{l}
{\left[G_{s} m_{p}\left(2 m_{u}+m_{d}\right)\right]} \\
+\left[G_{s} m_{n}\left(m_{u}+2 m_{d}\right)\right]
\end{array}\right\}
$$

\section{Two new coefficients and their applications}

In our earlier publications, in a quantum gravitational approach [9], we have introduced two coefficients 0.00189 and 0.006423 pertaining to nuclear stability and binding energy.

\section{A) Coefficient connected with Stability}

We noticed that,

1) As proton number increases, at stability zone, neutron number increases with square of the proton number.

2) Proportionality coefficient seems to be close to a number $0.0064[5,6,7]$. Quantitatively it can be fitted with a relation of the form,

$$
\begin{aligned}
& k \cong \alpha-\alpha \alpha_{s} \cong\left(1-\alpha_{s}\right) \alpha \cong 0.00644 \\
& \text { where } \alpha_{s} \cong 0.1181
\end{aligned}
$$

Based on the coefficient, $k \cong 0.00644$, neutron number close to stability zone can be expressed as,

$$
\left.\begin{array}{l}
N_{s} \cong Z+k Z^{2} \\
N_{s}-Z \cong k Z^{2}
\end{array}\right\}
$$

where, $Z=$ Proton number

$N_{s}=$ Neutron number close to stability

In terms of nucleon number,

$$
\left.\begin{array}{l}
A_{s} \cong 2 Z+k Z^{2} \\
A_{s}-2 Z \cong k Z^{2}
\end{array}\right\}
$$

where

$A_{s}=$ Nucleon number close to stability

$\left(N_{s}-Z\right) \cong\left(A_{s}-2 Z\right) \cong \Delta N_{s}$

$=$ Excess Neutron number close to stability zone

With this kind of relation, by guessing the, proton number, corresponding stable zone nucleon number can be estimated directly. With even-odd corrections and fine tuning the value of $k$, better understanding is possible. Considering $k \cong 0.00644$ and by considering a simple quadratic equation, relation (5) can be derived.

$$
\begin{gathered}
\text { Let, } X=\frac{Z k}{2} \\
\left.\begin{array}{c}
C=\frac{Z k}{2}=\frac{A k}{4}\left(\because \text { Initially, } Z=\frac{A}{2}\right) \\
\text { and } \quad X^{2}+X-C \cong 0 \\
\left\{\begin{array}{l}
X^{2} \text { coefficient }=1 \\
X \text { coefficient }=1
\end{array}\right\} \\
\rightarrow \frac{Z k}{2} \cong \frac{-1 \pm \sqrt{k A+1}}{2}
\end{array}\right\}
\end{gathered}
$$

With reference to observational data, it seems reasonable to assign the relation with mean stable mass number $\left(A_{m}\right)$.

$$
\left(A_{s}\right)_{\text {mean }} \cong A_{m} \cong 2 Z+k Z^{2}
$$

Best lower and upper limits for stable and relatively long living mass numbers can be approximated with the following relation.

$$
\begin{aligned}
& \left(A_{s}\right)_{l}^{u} \cong 2 Z+\left(1 \pm \alpha_{s}\right)^{2} k Z^{2} \\
& A_{l} \cong 2 Z+\left(1-\alpha_{s}\right)^{2} k Z^{2} \cong 2 Z+\left(0.78 k Z^{2}\right) \\
& A_{u} \cong 2 Z+\left(1+\alpha_{s}\right)^{2} k Z^{2} \cong 2 Z+\left(1.25 k Z^{2}\right)
\end{aligned}
$$

Using these relations as guidelines, long living isotopes of super heavy elements can be estimated.

With reference to the famous stability relation pertaining to semi empirical mass formula $[10,11,12]$,

$$
Z \cong \frac{A}{\left(2.0+\left(a_{c} / 2 a_{a}\right) A^{2 / 3}\right)} \cong \frac{A}{\left(2.0+0.0153 A^{2 / 3}\right)}
$$

where $a_{c} \cong 0.71 \mathrm{MeV}$ and $a_{a} \cong 23.2 \mathrm{MeV}$ 
Based on the proposed quadratic relation (6) and relation(9), it is possible to show that,

$$
Z \cong \frac{\sqrt{k A+1}-1}{k}
$$

Comparing relations (9) and (10), solution seems to be a relation of the form,

$$
0.0153 A^{2 / 3} \approx \sqrt{k A+1}-1
$$

$$
\left.\begin{array}{l}
\text { 12A) } \quad Z \cong \frac{A}{\left(2.0+0.0153 A^{2 / 3}\right)} \\
\text { 12B) } \quad Z \cong \frac{A}{1+\sqrt{k A+1}}
\end{array}\right\}
$$

$[\sqrt{k A+1}-1]$ seems to be more appropriate than $\left[0.0153 A^{2 / 3}\right]$ and it needs further study. With a curiosity, we noticed that,

$$
\left(\alpha_{s}^{2}+\alpha_{s}^{3}+\alpha_{s}^{4}+\alpha_{s}^{5}+\ldots\right) \cong 0.0158 \approx \frac{a_{c}}{2 a_{a}} \approx 0.0153
$$

\begin{tabular}{|c|c|c|c|}
\hline \multicolumn{4}{|c|}{$\begin{array}{l}\text { Table 1: Proton number comparison estimated } \\
\text { with relations (12A) and (12B) }\end{array}$} \\
\hline $\begin{array}{c}\text { Mass } \\
\text { number }\end{array}$ & $\begin{array}{c}\text { Proton } \\
\text { number } \\
\text { estimated } \\
\text { with relation } \\
\text { (12A) }\end{array}$ & $\begin{array}{l}\text { Proton } \\
\text { number } \\
\text { estimated } \\
\text { with } \\
\text { relation } \\
\text { (12B) }\end{array}$ & $\begin{array}{l}\text { Difference } \\
\text { in } \\
\text { estimated } \\
\text { proton } \\
\text { number }\end{array}$ \\
\hline 340 & 124 & 122 & 2 \\
\hline 332 & 121 & 120 & 1 \\
\hline 324 & 119 & 118 & 1 \\
\hline 316 & 117 & 115 & 2 \\
\hline 308 & 114 & 113 & 1 \\
\hline 300 & 112 & 111 & 1 \\
\hline 292 & 109 & 108 & 1 \\
\hline 284 & 107 & 106 & 1 \\
\hline 276 & 104 & 104 & 1 \\
\hline 268 & 102 & 101 & 1 \\
\hline 260 & 99 & 99 & 0 \\
\hline 252 & 97 & 96 & 1 \\
\hline 244 & 94 & 94 & 0 \\
\hline 236 & 91 & 91 & 0 \\
\hline
\end{tabular}

\begin{tabular}{|c|c|c|c|}
\hline 228 & 89 & 89 & 0 \\
\hline 220 & 86 & 86 & 0 \\
\hline 212 & 83 & 84 & -1 \\
\hline 204 & 81 & 81 & 0 \\
\hline 196 & 78 & 78 & 0 \\
\hline 188 & 75 & 76 & -1 \\
\hline 180 & 72 & 73 & -1 \\
\hline 172 & 70 & 70 & 0 \\
\hline 164 & 67 & 67 & 0 \\
\hline 156 & 64 & 65 & -1 \\
\hline 148 & 61 & 62 & -1 \\
\hline 140 & 58 & 59 & -1 \\
\hline 132 & 55 & 56 & -1 \\
\hline 124 & 52 & 53 & -1 \\
\hline 116 & 49 & 50 & -1 \\
\hline 108 & 46 & 47 & -1 \\
\hline 100 & 43 & 44 & -1 \\
\hline 92 & 40 & 41 & -1 \\
\hline 84 & 37 & 37 & 0 \\
\hline 76 & 33 & 34 & -1 \\
\hline 68 & 30 & 31 & -1 \\
\hline 60 & 27 & 28 & -1 \\
\hline 52 & 23 & 24 & -1 \\
\hline 44 & 20 & 21 & -1 \\
\hline 36 & 17 & 17 & 0 \\
\hline 28 & 13 & 13 & 0 \\
\hline 20 & 9 & 10 & -1 \\
\hline 12 & 6 & 6 & 0 \\
\hline 4 & 2 & 2 & 0 \\
\hline
\end{tabular}

where $a_{c} \cong 0.71 \mathrm{MeV}$ and $a_{a} \cong 23.2 \mathrm{MeV}$

See Table 1 for a comparison for estimated proton number starting from $\mathrm{A}=340$ to 4 .
Based on the data presented in Table 1, workability of the proposed coefficient $k \cong 0.00644$, can be validated.

\section{B) Coefficient connected with free nucleons}

With reference to our experience in this field, we understood that,

1) All the nucleons are not involving in nuclear binding energy scheme.

2) Nucleons that are not involving in nuclear binding energy scheme can be called as 'free nucleons'.

3) Number of free nucleons increases with increasing $\sqrt{Z N}$.

4) Nucleons that involve in nuclear binding energy scheme can be called as 'active nucleons'.

5) In finding the free nucleon number, with trialerror solutions, we are able to come across a number close to 0.00189 [9]. Quantitatively it can be fitted with a relation of the form, 


$$
\begin{aligned}
& f \cong\left(m_{d} / m_{u}\right) \alpha \alpha_{s} \cong 0.001863 \\
& \text { where } \alpha_{s} \cong 0.1181
\end{aligned}
$$

6) To a very good approximation, free nucleon number can be expressed with a relation of the form,

$$
A_{f} \cong f \sqrt{Z N} \cong 0.001863 \sqrt{Z N}
$$

7) Active nucleon number can be expressed with a relation of the form,

$$
A_{a} \cong A-A_{f} \cong A(1-0.001863 \sqrt{Z N})
$$

\section{Proposed nuclear binding energy scheme}

We propose that.

1) Nuclear binding energy $[13,14,15]$ can be understood with single energy coefficient and three simple terms.

2) Nuclear binding energy decreases with increasing number of free nucleons.

3) Nuclear binding energy increases with increasing number of active nucleons.

4) Nuclear binding energy decreases with increasing radius.

5) Mean stable mass number plays an interesting role in estimating the binding energy of other stable and unstable isotopes.

Based on these points, for estimating nuclear binding energy, we propose the following semi empirical relation for $\mathrm{Z}=3$ to 118 .

$$
\begin{gathered}
(B E)_{(z, A)} \cong\left\{A_{a}-A^{1 / 3}-\left(1+\frac{\left(A_{m}-A\right)^{2}}{A_{m}}\right)\right\} B_{0} \\
\left.\cong\left\{(1-0.001863 \sqrt{Z N}) A-A^{1 / 3}-\left(1+\frac{\left(A_{m}-A\right)^{2}}{A_{m}}\right)\right\} 10.1 \mathrm{MeV}\right\} \\
\text { where } B_{0} \cong \frac{G_{s} m_{p}\left(2 m_{u}+m_{d}\right)}{R_{0}}+\frac{G_{s} m_{n}\left(m_{u}+2 m_{d}\right)}{R_{0}} \\
\cong \frac{3}{2}\left(m_{u}+m_{d}\right) c^{2} \cong 10.245 \mathrm{MeV} \approx 10.1 \mathrm{MeV} \\
\text { and } R_{0} \cong \frac{2 G_{s} m_{p}}{c^{2}} \cong \frac{2 G_{s} m_{n}}{c^{2}} \cong 1.24 \mathrm{fm} .
\end{gathered}
$$

In this relation (17),

$$
\begin{aligned}
& \text { First term: }+(1-0.001863 \sqrt{Z N}) A \times 10.1 \mathrm{MeV} \\
& \text { Second term: } \quad-A^{1 / 3} \times 10.1 \mathrm{MeV} \\
& \text { Third term: } \quad-\left(1+\frac{\left(A_{m}-A\right)^{2}}{A_{m}}\right) \times 10.1 \mathrm{MeV}
\end{aligned}
$$


We are still working on understanding the physical significance of the third term $[16,17,18]$ and it needs in-depth study. Close to mean stable mass number,

$$
\begin{aligned}
& (B E)_{\left(z, A_{m}\right)} \cong\left\{\left(1-f \sqrt{Z N_{m}}\right) A_{m}-A_{m}^{1 / 3}-1\right\} 10.1 \mathrm{MeV} \\
& \text { where }\left\{\begin{array}{l}
A_{m} \cong\left(2 Z+k Z^{2}\right) \text { and } \\
N_{m} \cong A_{m}-Z \cong\left(Z+k Z^{2}\right)
\end{array}\right.
\end{aligned}
$$

See Table 2 nuclear binding energy of isotopes of $Z=50$ estimated with relations (7) and (17) compared with standard semi empirical mass formulae.

Note: To validate the relations (7) and (17), readers are encouraged to see the supplementary file for the whole range of nuclides starting from $Z=3$ to 120 . (Table 3 )

$\begin{aligned} & \text { In table 2, (Column-6)-SEMF - } \mathbf{1 ~ B E} \\ & \text { (https://en.wikipedia.org/wiki/Semi-empirical_mass_formula })\end{aligned}$
$B E \cong\left(a_{v}^{*} A\right)-\left(a_{s} * A^{2 / 3}\right)-\left(a_{c} * \frac{Z *(Z-1)}{A^{1 / 3}}\right)-\left(a_{a} * \frac{(A-2 Z)^{2}}{A}\right) \pm\left(\frac{a_{p}}{\sqrt{A}}\right)$
where $\left\{\begin{array}{l}a_{v} \cong 15.8 \mathrm{MeV} ; a_{s} \cong 18.3 \mathrm{MeV} ; a_{c} \cong 0.714 \mathrm{MeV} ; \\ a_{a} \cong 23.2 \mathrm{MeV} ; a_{p} \cong 12.0 \mathrm{MeV} ;\end{array}\right.$

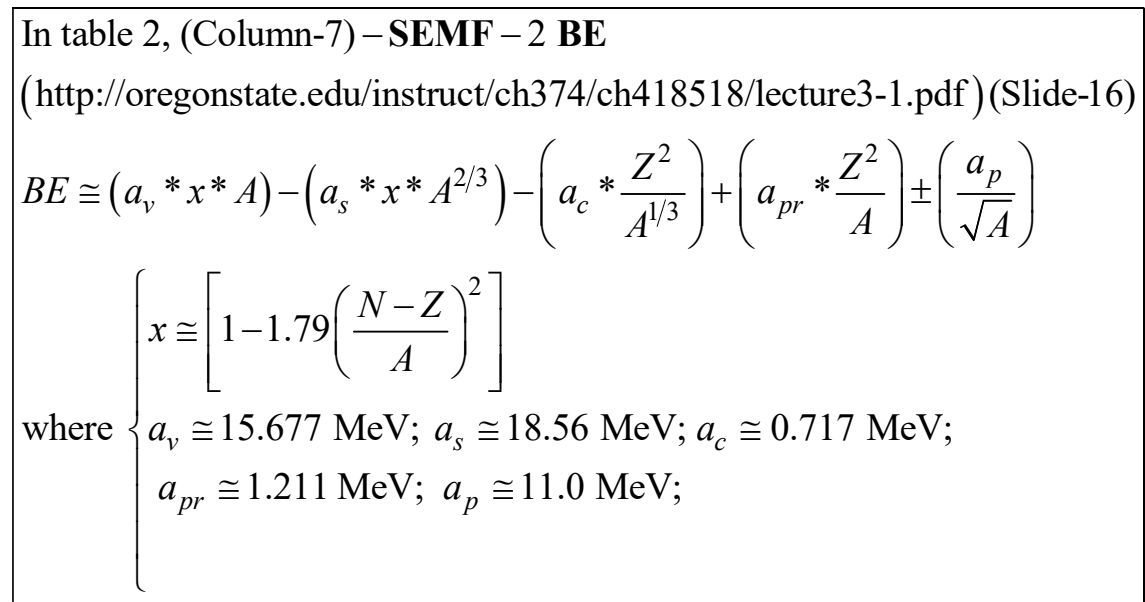

\begin{tabular}{|c|c|c|c|c|c|c|}
\hline \multicolumn{7}{|c|}{ Table 2: Estimated binding energy of isotopes of Z=50 } \\
\hline$Z$ & A & N & $\begin{array}{c}\text { Estimated } \\
\text { A_mean }\end{array}$ & $\begin{array}{c}\text { Estimated BE } \\
(\mathrm{MeV})\end{array}$ & $\begin{array}{c}\text { SEMF-1 BE } \\
(\mathrm{MeV})\end{array}$ & $\begin{array}{c}\text { SEMF-2 BE } \\
(\mathrm{MeV})\end{array}$ \\
\hline 50 & 100 & 50 & 116 & 836.6 & 810.1 & 813.0 \\
\hline 50 & 101 & 51 & 116 & 847.4 & 823.1 & 825.7 \\
\hline 50 & 102 & 52 & 116 & 858.0 & 838.0 & 840.2 \\
\hline
\end{tabular}




\begin{tabular}{|c|c|c|c|c|c|c|}
\hline 50 & 103 & 53 & 116 & 868.4 & 850.1 & 852.1 \\
\hline 50 & 104 & 54 & 116 & 878.6 & 864.1 & 865.7 \\
\hline 50 & 105 & 55 & 116 & 888.6 & 875.4 & 876.9 \\
\hline 50 & 106 & 56 & 116 & 898.5 & 888.6 & 889.8 \\
\hline 50 & 107 & 57 & 116 & 908.1 & 899.1 & 900.2 \\
\hline 50 & 108 & 58 & 116 & 917.6 & 911.5 & 912.4 \\
\hline 50 & 109 & 59 & 116 & 926.9 & 921.2 & 922.1 \\
\hline 50 & 110 & 60 & 116 & 936.0 & 932.8 & 933.6 \\
\hline 50 & 111 & 61 & 116 & 944.9 & 941.9 & 942.7 \\
\hline 50 & 112 & 62 & 116 & 953.7 & 952.8 & 953.6 \\
\hline 50 & 113 & 63 & 116 & 962.3 & 961.1 & 962.0 \\
\hline 50 & 114 & 64 & 116 & 970.6 & 971.4 & 972.3 \\
\hline 50 & 115 & 65 & 116 & 978.8 & 979.1 & 980.1 \\
\hline 50 & 116 & 66 & 116 & 986.9 & 988.8 & 989.8 \\
\hline 50 & 117 & 67 & 116 & 994.7 & 995.9 & 997.1 \\
\hline 50 & 118 & 68 & 116 & 1002.4 & 1004.9 & 1006.1 \\
\hline 50 & 119 & 69 & 116 & 1009.8 & 1011.4 & 1012.9 \\
\hline 50 & 120 & 70 & 116 & 1017.1 & 1019.9 & 1021.4 \\
\hline 50 & 121 & 71 & 116 & 1024.2 & 1025.9 & 1027.7 \\
\hline 50 & 122 & 72 & 116 & 1031.1 & 1033.8 & 1035.7 \\
\hline 50 & 123 & 73 & 116 & 1037.9 & 1039.3 & 1041.5 \\
\hline 50 & 124 & 74 & 116 & 1044.4 & 1046.7 & 1049.0 \\
\hline 50 & 125 & 75 & 116 & 1050.8 & 1051.6 & 1054.3 \\
\hline 50 & 126 & 76 & 116 & 1057.0 & 1058.5 & 1061.3 \\
\hline 50 & 127 & 77 & 116 & 1063.0 & 1063.1 & 1066.1 \\
\hline 50 & 128 & 78 & 116 & 1068.9 & 1069.5 & 1072.7 \\
\hline 50 & 129 & 79 & 116 & 1074.5 & 1073.5 & 1077.1 \\
\hline 50 & 130 & 80 & 116 & 1080.0 & 1079.5 & 1083.3 \\
\hline 50 & 131 & 81 & 116 & 1085.3 & 1083.2 & 1087.3 \\
\hline 50 & 132 & 82 & 116 & 1090.4 & 1088.7 & 1093.0 \\
\hline 50 & 133 & 83 & 116 & 1095.3 & 1091.9 & 1096.6 \\
\hline 50 & 134 & 84 & 116 & 1100.0 & 1097.0 & 1101.9 \\
\hline 50 & 135 & 85 & 116 & 1104.6 & 1099.9 & 1105.1 \\
\hline 50 & 136 & 86 & 116 & 1108.9 & 1104.6 & 1110.0 \\
\hline 50 & 137 & 87 & 116 & 1113.1 & 1107.1 & 1112.9 \\
\hline 50 & 138 & 88 & 116 & 1117.1 & 1111.5 & 1117.4 \\
\hline 50 & 139 & 89 & 116 & 1121.0 & 1113.6 & 1119.9 \\
\hline 50 & 140 & 90 & 116 & 1124.6 & 1117.6 & 1124.1 \\
\hline 50 & 141 & 91 & 116 & 1128.1 & 1119.4 & 1126.3 \\
\hline 50 & 142 & 92 & 116 & 1131.3 & 1123.0 & 1130.1 \\
\hline 50 & 143 & 93 & 116 & 1134.4 & 1124.5 & 1132.0 \\
\hline
\end{tabular}




\begin{tabular}{|l|l|l|l|l|l|l|}
\hline 50 & 144 & 94 & 116 & 1137.3 & 1127.8 & 1135.5 \\
\hline 50 & 145 & 95 & 116 & 1140.1 & 1129.0 & 1137.0 \\
\hline 50 & 146 & 96 & 116 & 1142.6 & 1131.9 & 1140.2 \\
\hline 50 & 147 & 97 & 116 & 1145.0 & 1132.8 & 1141.4 \\
\hline 50 & 148 & 98 & 116 & 1147.2 & 1135.5 & 1144.3 \\
\hline 50 & 149 & 99 & 116 & 1149.2 & 1136.1 & 1145.2 \\
\hline 50 & 150 & 100 & 116 & 1151.0 & 1138.5 & 1147.8 \\
\hline 50 & 151 & 101 & 116 & 1152.7 & 1138.8 & 1148.5 \\
\hline 50 & 152 & 102 & 116 & 1154.1 & 1140.9 & 1150.8 \\
\hline 50 & 153 & 103 & 116 & 1155.4 & 1140.9 & 1151.1 \\
\hline 50 & 154 & 104 & 116 & 1156.5 & 1142.8 & 1153.2 \\
\hline 50 & 155 & 105 & 116 & 1157.4 & 1142.5 & 1153.3 \\
\hline 50 & 156 & 106 & 116 & 1158.1 & 1144.1 & 1155.0 \\
\hline 50 & 157 & 107 & 116 & 1158.7 & 1143.7 & 1154.9 \\
\hline 50 & 158 & 108 & 116 & 1159.0 & 1145.0 & 1156.4 \\
\hline 50 & 159 & 109 & 116 & 1159.2 & 1144.3 & 1156.0 \\
\hline 50 & 160 & 110 & 116 & 1159.2 & 1145.4 & 1157.2 \\
\hline
\end{tabular}

\section{Conclusion}

With reference to the data presented in Tables 1 and 2 , our proposed concepts and relations can be recommended for further investigation. By refining the values of $\left(G_{s}, m_{u}, m_{d}\right)$, magnitudes of $\left(\alpha_{s}, f, k, B_{0}\right)$ can be refined.

\section{Acknowledgements}

Author Seshavatharam is indebted to professors shri M. Nagaphani Sarma, Chairman, shri K.V. Krishna Murthy, founder Chairman, Institute of Scientific Research in Vedas (I-SERVE), Hyderabad, India and Shri K.V.R.S. Murthy, former scientist IICT (CSIR), Govt. of India, Director, Research and Development, I-SERVE, for their valuable guidance and great support in developing this subject.

\section{References}

[1] Ghahramany $\mathrm{N}$ et al. New scheme of nuclide and nuclear binding energy from quark-like model. Iranian Journal of Science \& Technology. A3: 201-208 (2011)

[2] Ghahramany $\mathrm{N}$ et al. New approach to nuclear binding energy in integrated nuclear model. Journal of Theoretical and Applied Physics. 6:3 (2012)

[3] M. Tanabashi et al. (Particle Data Group), Phys.
Rev. D 98, 030001 (2018)

[4] K. Tennakone, Electron, muon, proton, and strong gravity. Phys. Rev. D, 10, 1722 (1974)

[5] C. Sivaram and K. Sinha, Strong gravity, black holes, and hadrons. Physical Review D., 16(6), 1975-1978 (1977)

[6] De Sabbata V and M. Gasperini. Strong gravity and weak interactions. Gen. Relat. Gravit. 10, 9, 731-741, (1979)

[7] Salam A, Sivaram C. Strong Gravity Approach to QCD and Confinement. Mod. Phys. Lett., v. A8(4), 321-326 (1993)

[8] Roberto Onofrio. On weak interactions as shortdistance manifestations of gravity. Modern Physics Letters A 28, 1350022 (2013)

[9] Seshavatharam UVS and Lakshminarayana S. On the Role of Squared Neutron Number in Reducing Nuclear Binding Energy in the Light of Electromagnetic, Weak and Nuclear Gravitational Constants - A Review. Asian Journal of Research and Reviews in Physics, 2(3): 1-22, (2019)

[10] Roy Chowdhury P et al. Modified BetheWeizsacker mass formula with isotonic shift and new driplines. Mod. Phys. Lett. A20:16051618 (2005)

[11] W. Zhang et al. Magic numbers for super heavy nuclei in relativistic continuum HartreeBogoliubov theory, Nuclear Physics A, Volume 753, 1-2, 106-135 (2005)

[12] A. Bohr and B. R. Mottelson, Nuclear Structure Vol. 1 (W. A. Benjamin Inc., New York, 
Amesterdam, 1969).

[13] Cht. Mavrodiev S, Deliyergiyev MA. Modification of the nuclear landscape in the inverse problem framework using the generalized Bethe-Weizsäcker mass formula. Int. J. Mod. Phys. E27: 1850015 (2018)

[14] Xiaa XW, et al. The limits of the nuclear landscape explored by the relativistic continuum Hatree-Bogoliubov theory. Atomic Data and Nuclear Data Tables. 121-122: 1-215 (2018)

[15] P. Mollera, A. J. Sierka, T. Ichikawab, H. Sagawa. Nuclear ground-state masses and deformations: FRDM(2012). Atomic Data and Nuclear Data Tables. Vol.109-110: 1-204 (2016)
[16] Seshavatharam UVS and Lakshminarayana S. On the Role of Large Nuclear Gravity in Understanding Strong Coupling Constant, Nuclear Stability Range, Binding Energy of Isotopes and Magic proton numbers - A Critical Review. J. Nucl. Phys. Mat. Sci. Rad. A. 6(2), 142-160 (2019)

[17] Seshavatharam UVS and Lakshminarayana S. On the role of four gravitational constants in nuclear structure. Mapana Journal of Sciences, 18(1), 2145 (2019)

[18] Seshavatharam UVS and Lakshminarayana S. Implications and Applications of Fermi Scale Quantum Gravity. Preprints, 2019110134 (doi: 10.20944/preprints201911.0134.v1 (2019) 